\title{
Vasopressin and Vasopressin Receptor Antagonists
}

\author{
Yun Kyu Oh, M.D.
}

\author{
Department of Internal Medicine, Seoul National University Boramae Hospital \\ Department of Internal Medicine, Seoul National University College of Medicine, Seoul, Korea
}

\begin{abstract}
Vasopressin, a neurohypophyseal peptide hormone, is the endogenous agonist at V1a, V1b, and V2 receptors. The most important physiological function of vasopressin is the maintenance of water homeostasis through interaction with V2 receptors in the kidney. Vasopressin binds to V2 receptor and increases the number of aquaporin-2 at the apical plasma membrane of collecting duct principal cells. That induces high water permeability across the membrane. Several non-peptide vasopressin receptor antagonists have been developed and are being studied primarily for treating conditions characterized by hyponatremia and fluid overload. Further studies are needed to determine how they are best used in these situations.
\end{abstract}

Key Words : vasopressins; aquaporin 2; hyponatremia; vasopressin receptor antagonist

\section{Introduction}

Vasopressin, the antidiuretic hormone, is the major physiologic regulator of renal water excretion and plays an important role in regulating serum osmolality. Several clinical conditions have been associated with inappropriately increased vasopressin secretion that causes antidiuresis, with subsequent retention of water by the kidney. The impaired renal water excretion results in increasing total body water, causing hypo-osmolality and hyponatremia.

\section{Vasopressin synthesis and regulation of secretion}

Vasopressin is a nonapeptide (molecular weight 1,099) synthesized in the paraventricular and supraoptic nuclei of the hypothalamus (Fig. 1). The hormone is transported to the posterior pituitary gland and stored. It is released to the bloodstream upon appropriate stimulation. The most important mechanism involved in the control of the release

Received January 1, 2008. Accepted February 1, 2008.

Corresponding author: Yun Kyu Oh, M.D.

Department of Internal Medicine, Seoul National University Boramae

Hospital, Seoul National University College of Medicine,

425 Shindaebang 2-dong, Dongjak-gu, Seoul, 156-707, Korea

Tel : +82-2-840-2821, Fax: +82-2-831-2826

E-mail: yoonkyu@snu.ac.kr of vasopressin is the effective osmotic pressure of plasma. Changes of as little as $1 \%$ in plasma osmolality can cause significant increases in plasma vasopressin levels with proportional increases in urine concentration. Maximal antidiuresis is achieved after increases in plasma osmolality of only $2-4 \%$ above the threshold for vasopressin secretion. In addition, the rapid response of pituitary vasopressin secretion and its short half life enable adjustment of renal water excretion to changes in plasma osmolality on a minute-to-minute basis.

Hypovolemia which is detected by arterial and atrial baroreceptors is also a potent stimulus for vasopressin

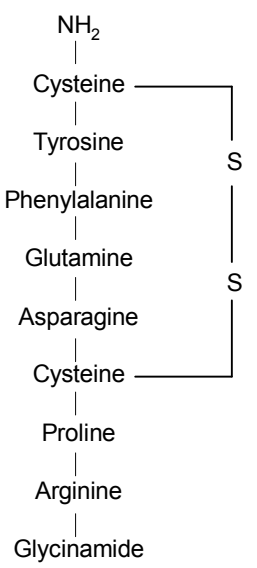

Fig. 1. Structures of arginine vasopressin. 
secretion. Although the osmotic mechanism of regulation of vasopressin secretion is very sensitive, a $15-20 \%$ fall in blood pressure is required to induce maximal antidiuresis. Vasopressin release can be influenced by a variety of other factors that are not directly related to osmolal or volume balance. Nausea, hypoglycemia, angiotensin II, nonspecific stress, and acute hypoxia or hypercapnia stimulate vasopressin secretion, whereas drinking lowers plasma vasopressin before there is any appreciable decrease in plasma osmolality. A variety of drugs nicotine, also stimulate or inhibit vasopressin secretion ${ }^{1)}$.

\section{Vasopressin receptors}

V1a, V2, and V1b (or V3) vasopressin receptors have been identified. The receptors are members of the Gprotein coupled receptor superfamily. Activation of V1a receptors located in vascular smooth muscle cells, myocardium, hepatocyte, platelets, and myometrium results in vasoconstriction, myocardial hypertrophy, hepatic glycogenolysis, platelet aggregation, and uterine contraction. $\mathrm{V} 1 \mathrm{~b}$ receptors are mainly located in the anterior pituitary and regulate the secretion of adrenocorticotropic hormone (ACTH). The V2 receptors (V2R), present in the basolateral membrane of the principal cells of the renal collecting tubules and connecting tubules, mediate the osmotic effect of vasopressin ${ }^{2)}$. The V2R are also present in thick ascending limbs of the loop of Henle and stimulate $\mathrm{NaCl}$ reabsorption into the medullary interstitium ${ }^{3)}$. They are found in vascular endothelium and effect release of the von Willebrand factor and Factor VIII (Table 1).

\section{The aquaporins}

The V2R is a $41 \mathrm{KDa}$ protein of 371 residues with seven transmembrane domains. It is expressed on the plasma membrane of collecting duct principal cells. Binding of vasopressin to the $\mathrm{V} 2 \mathrm{R}$ activates the stimulatory heterotrimeric GTP-binding protein (Gs). The activated Gs then stimulates adenylate cyclase, resulting in an increase in intracellular cAMP. The latter activates protein kinase A, which phosphorylates preformed aquaporin-2 (AQP2) water channels located in intracellular vesicles. Phosphorylation of AQP2 promotes trafficking to the apical membrane, followed by exocytic insertion of AQP2 vesicles into the cell membrane. This is the rate limiting step for transepithelial water flow. Aquaporins-3 and -4 (AQP3 and AQP4) are constitutively present in the basolateral membrane. However, AQP3 is only regulated by vasopressin. AQP3 expression changes following vasopressin stimulation and several pathologic states. When the effect of vasopressin is dissipated, the water channels are removed from the apical membrane by endocytosis and returned to the cytoplasm. AQP2 expression levels markedly increase in response to long term dehydration, with an increased abundance of AQP2 in the apical plasma membrane. Therefore, collecting duct water permeability is regulated by short-term and long-term mechanisms, both critically involving $\mathrm{AQP} 2^{4,5)}$ (Fig. 2).

\section{Role of vasopressin in hyponatremic states}

Plasma vasopressin levels have been found to be in-

Table 1. Vasopressin Receptor Subtypes

\begin{tabular}{lll}
\hline Receptor & Localization & Function \\
\hline V1a & Vascular smooth muscle & Vasoconstriction, myocardial hypertrophy \\
& Platelets & Platelets aggregation \\
& Hepatocytes & Glycogenolysis \\
& Myometrium & Uterine contraction \\
V1b (V3) & Anterior pituitary & ACTH release \\
V2 & Basolateral membrane of collecting tubule & Insertion of AQP2 water channels into apical membrane, induction of \\
& & AQP2 synthesis \\
& Vascular endothelium & vWF and Factor VIII release \\
& Vascular smooth muscle & Vasodilation \\
\hline
\end{tabular}

$\mathrm{AQP}$, aquaporin-2; vWF, von Willebrand factor; $\mathrm{ACTH}$, adrenocorticotropic hormone. 
appropriately elevated in several conditions such as congestive heart failure (CHF), syndrome of inappropriate antidiuretic hormone secretion (SIADH), liver cirrhosis (LC), polycystic kidney disease, nephrotic syndrome, and surgical stress. In SIADH, vasopressin release is not fully suppressed despite hypotonicity. In CHF and LC, a diminished glomerular filtration rate or impaired delivery of solute to the diluting segment impair maximal water excretory capacity. However, the persistence of vasopressin release due to nonosmotic stimuli is predominantly responsible for water retention in these disorders ${ }^{6}$.

\section{Vasopressin receptor antagonists}

Vasopressin receptor antagonists (VRAs) was first developed as a peptide antagonist during the 1970s. Although a peptide mediate V2R antagonist effect in animals, a phase 1 trial showed that the agent had a paradoxical weak V2R agonist in human studies. Thus, further development of peptide antagonists was abandoned ${ }^{7)}$. Using a functional screening strategy, non-peptide VRAs were subsequently identified. The four non-peptide agents, include conivaptan, lixivaptan, tolvaptan, and satavaptan, are now in various stages of clinical trials (Table 2). Molecular modeling of binding sites suggests that these antagonists penetrate deeper into the transmembrane region of the V2R than vasopressin. They thereby prevent binding of native hormone ${ }^{8)}$.

Conivaptan is a combined V1aR and V2R antagonist, whereas others are selective V2R antagonists. All Agents of this class are inhibitors of the cytochrome P350 3A4 system, but conivaptan is the most potent. In December 2005, the US FDA approved conivaptan for treatment of euvolemic hyponatremia. Although the drug is orally active, to minimize the possibility of drug interaction, the FDA has restricted its distribution to a parenteral form for short-term in-hospital use only. Other VRAs are being

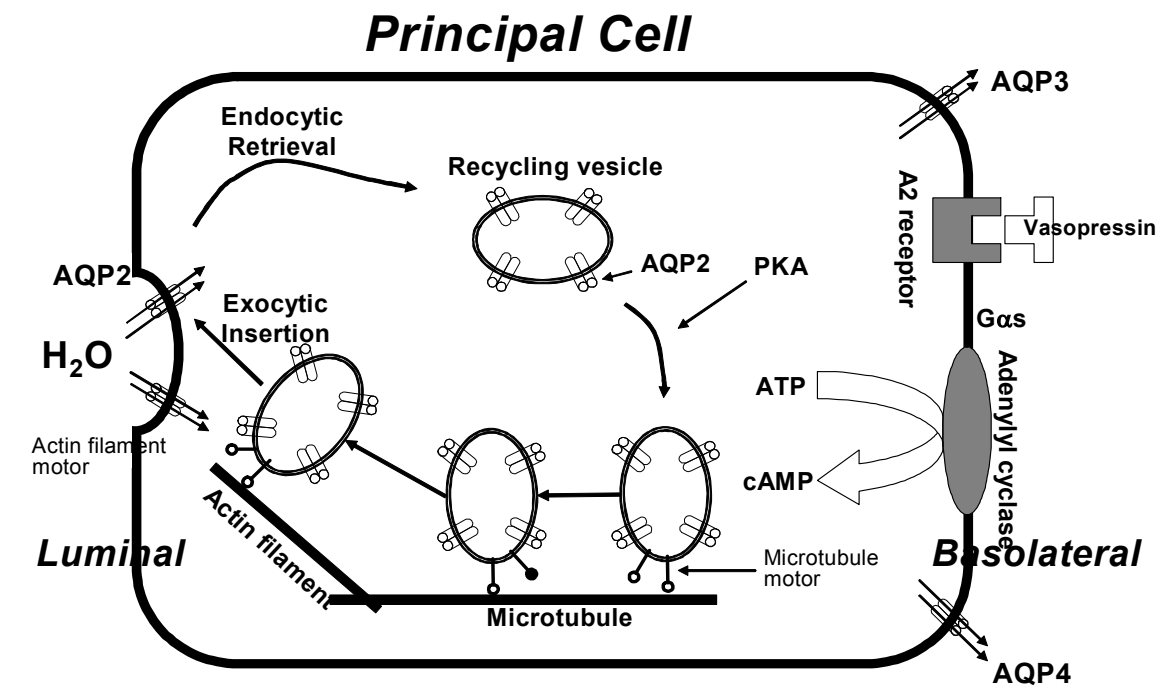

Fig. 2. Schematic representation of some major events that result from the interaction of vasopressin with its V2 vasopressin receptor on the basolateral plasma membrane of collecting duct principal cells. AQP, aquaporin; Gas, G protein $\alpha$ subunit; PKA, protein kinase A; cAMP, cyclic adenosine 3',5'-monophosphate.

Table 2. Non-Peptide VRAs under Commercial Development

\begin{tabular}{lccl}
\hline Compound & Receptor & Route & Manufacturer \\
\hline Conivaptan (YM-087) & V1a+V2 & i.v. & Astellas (Tokyo, Japan) \\
Lixivaptan (VPA-985) & V2 & Oral & CardioKine (Philadelphia, PA, USA) \\
Tolvaptan (OPC-41061) & V2 & Oral & Otsuka (Tokyo, Japan) \\
Satavaptan (SR-121463B) & V2 & Oral & Sanofi-Aventis (Paris, France) \\
\hline
\end{tabular}

VRAs, Vasopressin receptor antagonists. 
developed for long-term oral use ${ }^{9)}$.

\section{Potential uses for vasopressin receptor antagonists}

\section{Hyponatremia}

In many clinical trials, these agents produce an aquaresis leading to increased plasma $\mathrm{Na}^{+}$concentration in the majority patients with hyponatremia due to SIADH, CHF, and LC. Although the FDA approved conivaptan only for the indication of euvolemic hyponatremia, increased exposure of larger numbers of clinical trials should lead to approval for the indication of hypervolemic hyponatremia. The optimum use of VRAs has not yet been determined, but some predictions can be made according to clinical trials. For hyponatremia in hospitalized patients, parenteral conivaptan will likely be the preferred agents. In patients for whom oral therapy is suitable and for chronic forms of hyponatremia, selective V2R antagonists such as lixivaptan, tolvaptan, and satavaptan will likely be useful.

Safety issues must also be considered carefully. The possibility of ovecorrection has been significantly concerned, but osmotic demyelination has not been reported in all of the clinical trials. Safeguard, such as avoidance of correction of the plasma sodium concentration at a rate faster than $8-12 \mathrm{mEq} / \mathrm{L} / 24 \mathrm{hr}$, will be sufficient to protect against overly rapid correction of plasma sodium concentration. A second concern is to avoid using VRAs in cases of hypovalemic hyponatremia, where an aquaresis would aggravate the underlying volume depletion. Hypotension, particularly with conivaptan, or bleeding complications should be carefully monitored ${ }^{9,10)}$.

\section{Congestive heart failure}

In the ACTIV in CHF (Acute and Chronic therapeutic Impact of a Vasopressn Antagonist in Congestive Heart Failure) trial, 319 hospitalized patients with heart failure exacerbation were randomized to tolvaptan or placebo. Worsening CHF or unscheduled visits for CHF within 60 days were not different among the treatment groups, although post-hoc analysis suggests that cardiovascular mortality may be reduced in the tolvaptan group in the higher risk group with kidney function impairment or severe congestive findings ${ }^{11)}$. Until well-powered studies, to determine whether VRA therapy will have beneficial effect on the CHF neurohormonal cascade have been completed, VRAs are not recommended in patients with CHF.

\section{Liver cirrhosis}

Because the presence of portal hypertension in these patients, V1aR blockade may produce hypotension and variceal bleeding owing to antagonism of vasopressin pressor effects in this vascular bed. Thus, only selective V2R antagonists can be recommended for these patients ${ }^{9}$.

\section{Other diseases}

A potential role for the VRAs in attenuating polyuria in nephrogenic diabetes insipidus and cyst development in polycystic kidney disease is being explored ${ }^{9)}$.

\section{Conclusion}

Vasopressin is a neuropeptide hormone that plays an important role in water metabolism via regulation of AQP2 in the principal cells of the renal collecting tubules and connecting tubules. The SIADH, CHF, and LC are among many conditions associated with abnormal water retention mediated by vasopressin release inappropriate to plasma osmolality. VRAs target the cause of abnormal water retention by producing an increase in electrolyte- free water excretion. Thus, these agents will provide highly specific therapy for euvolemic or hypervolemic hyponatremia, $\mathrm{CHF}$, and $\mathrm{LC}$.

\section{References}

1) Berl T, Verbalis J: Pathophysiology of water metabolism. In: Brenner \& Rector's the kidney, 7th ed., edited by Brenner BM, Rector FC, Philadelphia, Saunders, 2004, p857-919

2) Petersen MB: The effect of vasopressin and related compounds at V1a and V2 receptors in animal models relevant to human disease. Basic Clin Pharmacol Toxicol 99:96103, 2006

3) Hebert SC, Culpepper RM, Andreoli TE: $\mathrm{NaCl}$ transport in mouse medullary thick ascending limbs. II. ADH en- 
hancement of transcellular $\mathrm{NaCl}$ cotransport; origin of transepithelial voltage. Am J Physiol 241:F432-442, 1981

4) Nielsen S, Frokiaer J, Marples D, Kwon TH, Agre P, Knepper MA: Aquaporins in the kidney: from molecules to medicine. Physiol Rev 82:205-244, 2002

5) Sheikh-Hamad D, Marples D, Nielsen S: Cell biology of vasopressin and aquaporins. In: Acid-base and electrolyte disorders : a companion to Brenner \& Rector's the kidney, 1st ed., edited by DuBose TD, Hamm LL, Philadelphia, Saunders, 2002, p217-228

6) Guyton A: The body fluids and kidneys. In: Textbook of medical physiology, 11th ed., edited by Guyton AC, Hall JE, Philadelphia, Elsevier Saunders, 2006, p291-414

7) Verbalis JG: Vasopressin V2 receptor antagonists. J Mol Endocrinol 29:1-9, 2002
8) Macion-Dazard R, Callahan N, Xu Z, Wu N, Thibonnier M, Shoham M: Mapping the binding site of six nonpeptide antagonists to the human V2-renal vasopressin receptor. J Pharmacol Exp Ther 316:564-571, 2006

9) Greenberg A, Verbalis JG: Vasopressin receptor antagonists. Kidney Int 69:2124-2130, 2006

10) Ali F, Guglin M, Vaitkevicius $P$, Ghali JK: Therapeutic potential of vasopressin receptor antagonists. Drugs 67: 847-858, 2007

11) Gheorghiade $M$, Gattis WA, O'Connor CM, Adams KF, Jr., Elkayam U, Barbagelata A, et al.: Effects of tolvaptan, a vasopressin antagonist, in patients hospitalized with worsening heart failure: a randomized controlled trial. JAMA 291:1963-1971, 2004 